\title{
BMJ Open Exploring variation in human papillomavirus vaccination uptake in Switzerland: a multilevel spatial analysis of a national vaccination coverage survey
}

\author{
Maurane Riesen, ${ }^{1,2}$ Garyfallos Konstantinoudis, ${ }^{1,3}$ Phung Lang, ${ }^{4}$ Nicola Low, ${ }^{1}$ \\ Christoph Hatz, ${ }^{4}$ Mirjam Maeusezahl, ${ }^{5}$ Anne Spaar, ${ }^{5}$ Marc Bühlmann, ${ }^{6}$ \\ Ben D Spycher, ${ }^{1,7}$ Christian L Althaus ${ }^{1}$
}

To cite: Riesen $M$,

Konstantinoudis G, Lang P, et al. Exploring variation in human papillomavirus vaccination uptake in Switzerland: a multilevel spatial analysis of a national vaccination coverage survey. BMJ Open 2018;8:e021006. doi:10.1136/ bmjopen-2017-021006

- Prepublication history and additional material for this paper are available online. To view these files, please visit the journal online (http://dx.doi. org/10.1136/bmjopen-2017021006).

BDS and CLA contributed equally.

MR and GK contributed equally.

Received 13 December 2017 Revised 8 February 2018 Accepted 2 March 2018

Check for updates

For numbered affiliations see end of article.

Correspondence to

Maurane Riesen;

maurane.riesen@ispm.unibe.ch

\section{ABSTRACT}

Objective Understanding the factors that influence human papillomavirus (HPV) vaccination uptake is critically important to the design of effective vaccination programmes. In Switzerland, HPV vaccination uptake ( $\geq 1$ dose) by age 16 years among women ranges from $31 \%$ to $80 \%$ across 26 cantons (states). Our objective was to identify factors that are associated with the spatial variation in HPV vaccination uptake.

Methods We used cross-sectional data from the Swiss National Vaccination Coverage Survey 2009-2016 on HPV vaccination status ( $\geq 1$ dose) of 14-17-year-old girls, their municipality of residence and their nationality for 21 of 26 cantons ( $n=8965)$. We examined covariates at municipality level: language, degree of urbanisation, socioeconomic position, religious denomination, results of a vote about vaccination laws as a proxy for vaccine scepticism and, at cantonal level, availability of school-based vaccination and survey period. We used a series of conditional autoregressive models to assess the effects of covariates while accounting for variability between cantons and municipal-level spatial autocorrelation.

Results In the best-fit model, living in cantons that have school-based vaccination (adjusted OR 2.51; 95\% credible interval 1.77 to 3.56 ) was associated with increased uptake, while living in municipalities with lower acceptance of vaccination laws was associated with lower HPV vaccination uptake (OR $0.61 ; 95 \%$ credible interval 0.50 to 0.73 ). Overall, the covariates explained $88 \%$ of the municipal-level variation in uptake.

Conclusions In Switzerland, both cantons and community opinion about vaccination play a prominent role in the variation in HPV vaccination uptake. To increase uptake, efforts should be made to mitigate vaccination scepticism and to encourage school-based vaccination.

\section{INTRODUCTION}

Human papillomavirus (HPV) is the most common viral infection of the reproductive tract. ${ }^{1}$ Persistent infections with HPV types 16 and 18 are responsible for $70 \%$ of cervical

\section{Strengths and limitations of the study}

- We used individual-level data from a national vaccination coverage survey covering more than $90 \%$ of the Swiss population during the evolution of human papillomavirus vaccination programmes from 2009 to 2016 .

- We adjusted for spatial confounding using BesagYork-Mollié conditional autoregressive models.

- We used the outcome of a popular referendum against a revised epidemic law as a proxy measure of vaccine scepticism.

- The survey sampling methods and response rates differed across cantons (states).

- Five out of 26 cantons did not participate in the study.

cancers and precancerous cervical lesions. ${ }^{1}$ Genital HPV types also cause anogenital warts and cancers of the anus, vulva, vagina and penis. ${ }^{1}$ In 2006, the first vaccine against HPV was licensed and, by 2016, at least 68 countries had implemented vaccination programmes for the prevention of cervical cancer in at least one region. ${ }^{2}$ Optimal HPV vaccination coverage (full-dose schedule) is estimated to be around $70 \%$ for women but there are large geographical disparities in vaccination coverage between and within countries. $^{2}{ }^{3}$ The UK and Australia have reached homogeneous levels of around $70 \%$ vaccination coverage. ${ }^{4-6}$ In contrast, many countries including Italy, France, Switzerland, Germany, the Netherlands and the USA experience lower national coverage rates with large regional variations. ${ }^{7-11}$

There are several challenges to achieving high levels of HPV vaccine coverage, such as concerns that it might promote risky 
sexual behaviour in adolescents and logistical issues about reaching adolescents. ${ }^{12}$ Individuals shape the geographic and social contexts in which they live, but their behaviour is also affected by their context. ${ }^{13}$ An understanding of community-level and individual-level factors associated with HPV vaccination uptake is therefore important. It has been shown that countries with extensive school-based vaccination reached markedly higher uptake rates. ${ }^{14}$ Conversely, lower levels of HPV vaccine uptake have been found in communities or states with high levels of votes for religious or conservative parties, ${ }^{11} 15$ but it is not known how closely these reflect attitudes towards vaccination. At the individual level, findings about factors such as socioeconomic position (SEP), ethnicity or religious affiliation are more mixed. ${ }^{811}$ 16-22 For example, poverty, based on either low income or SEP, has been found to be associated with both lowerr116 19 20 23-26 and higher HPV vaccination uptake. ${ }^{18} 202127$ Furthermore, few studies have accounted for spatial autocorrelation due to unknown confounding. ${ }^{16} 1722$ Neglecting this spatial autocorrelation can lead to spurious associations. ${ }^{28}$

Switzerland provides a valuable setting for investigating regional differences in vaccine uptake. The country is spatially divided into 26 cantons (states) and four language regions (German, French, Italian and Romansh). The cantons have a high degree of autonomy with devolved administration of health and education. Within cantons, municipalities also enjoy a high level of autonomy including the power to pass municipal laws. People vote regularly in referendums on a wide range of issues that then determine legislation. All Swiss cantons implemented HPV vaccination programmes by the end of 2008, targeting 11-14-year-old girls for basic vaccination and additionally including young women and men (up to 26 years old) for complementary vaccination. A two-dose vaccination schedule (administered at 0 and 6 months) was introduced in 2012, replacing the earlier three-dose schedule. The HPV basic vaccination programmes for school-aged girls differ widely between cantons, ranging from the simple distribution of educational material, informing parents that vaccination is available, through to school-based vaccination delivery. ${ }^{29-31}$

The objective of this study was to investigate the spatial heterogeneity of HPV vaccination uptake in Switzerland, and to identify factors at different spatial levels that explain this variation. We investigated both political and cultural contextual factors. We hypothesised that the canton of residence would represent an important contextual factor influencing whether or not an individual girl had received HPV vaccination. We further expected that covariates at the level of the municipality, such as the degree of political scepticism about vaccination, socioeconomic status, language, religion or the level of urbanisation, could represent important contextual factors that play a role in explaining differences in uptake.

\section{METHODS}

We conducted a multilevel spatial analysis of the Swiss National Vaccination Coverage Survey (SNVCS). ${ }^{32-34}$ We used a series of Bayesian hierarchical logistic regression models that include spatial autocorrelation, a random effect to account for variability between the cantons, and several covariates.

\section{Individual-level data}

We used data from the SNVCS, which is a national cross-sectional survey that monitors immunisation coverage of children and adolescents. ${ }^{32-34}$ This survey has been designed to be representative of the Swiss population with respect to key demographic characteristics. ${ }^{34}$ The Swiss Federal Office of Public Health mandates the Epidemiology, Biostatistics and Prevention Institute (EPBI, University of Zurich, Switzerland) to collate data in 3-year cycles from all cantons in surveys organised either by EBPI or by the individual cantons. Three different cross-sectional sampling methods were used: cluster sampling (municipalities), simple random sampling or information collection by school nurses (see online supplementary table 1 ). The methodology is described in detail elsewhere. ${ }^{34}$ For cluster sampling and simple random sampling, the parents of 16-year-old girls received an invitation (by email or by phone) and were asked to send a copy of the daughter's vaccination card. In three cantons, school nurses recorded the information at health checkup days for 14-16-year-old girls. We used anonymised, individual-level information about HPV vaccination status (having received at least one dose of HPV vaccine), nationality (Swiss or non-Swiss, coded as 0 and 1, respectively) and municipality of residence. Hence, vaccination coverage refers to vaccination initiation and not the completed full-dose schedule. Missing individual information about nationality was replaced with the proportion of non-Swiss people in the subject's municipality of residence based on the national census in $2013 .^{35}$

\section{Covariates at municipality and cantonal level}

At the cantonal level, we considered survey period (2008-2010, 2011-2013, 2014-2016, excluding 2008 because not all cantons had implemented their vaccination programme at the time) and availability of schoolbased HPV vaccination delivery (defined as provision by at least one school in the canton, yes, no) ${ }^{36}$ At the municipality level, we considered language region (French, German or Italian), ${ }^{37}$ majority religious denomination ( $\geq 50 \%$ catholic, $\geq 50 \%$ protestant or neither), ${ }^{38} \mathrm{SEP}$ (mean Swiss SEP, a neighbourhood-based measure of with lower values indicating lower $\mathrm{SEP}^{39}{ }^{40}$ ) and level of urbanisation (rural, semiurban, urban, based on standard Swiss classifications ${ }^{37}$ ).

We also considered the municipality-level results of a popular referendum in 2013 as a proxy measure of vaccine scepticism. ${ }^{41}{ }^{42}$ The referendum was proposed by opponents of a revision to a national law about the control of epidemics, which included new recommendations about 
Table 1 Comparison of Bayesian hierarchical logistic regression models that explain the spatial heterogeneity of HPV vaccination uptake in Switzerland

\begin{tabular}{|c|c|c|c|c|}
\hline Model type & $\begin{array}{l}\text { Spatial } \\
\text { component }\end{array}$ & $\begin{array}{l}\text { Cantonal } \\
\text { random } \\
\text { effect }\end{array}$ & Covariates* & DIC \\
\hline $\begin{array}{l}\text { Model } 1 \\
\text { (BYM unadjusted) }\end{array}$ & $x$ & & & 11513 \\
\hline $\begin{array}{l}\text { Model } 2 \\
\text { (BYM cantonal) }\end{array}$ & $x$ & $x$ & & 11489 \\
\hline Model 3 (full) & $x$ & $x$ & $x$ & 11417 \\
\hline Model 4 & $x$ & & $x$ & 11432 \\
\hline Model 5 & & $x$ & & 11541 \\
\hline Model 6 & & $x$ & $x$ & 11450 \\
\hline $\begin{array}{l}\text { Model } 7 \\
\text { (logit model) }\end{array}$ & & & $x$ & 11557 \\
\hline $\begin{array}{l}\text { Model } 8 \dagger \\
\text { (univariable) }\end{array}$ & & & $x$ & - \\
\hline
\end{tabular}

*Nationality, urbanisation level, Swiss socioeconomic position, political opinion, religious denominations, language region, schoolbased human papillomavirus vaccination programme, survey period.

†Model 8 (univariable) was adjusted for one variable at time. BYM, Besag-York-Mollié prior; DIC, deviance information criterion.

vaccination (see online supplementary material, section 1 , page 3). We calculated the percentage of votes in favour of revision of the law for each municipality. We considered municipalities with a low percentage of people in favour of the revised law as having higher proportions of people who are sceptical about vaccination than municipalities that strongly favoured the revised law. For the continuous variables, referendum results and the Swiss SEP index, we generated quartiles and compared the lowest and the highest quartiles with the second and third (baseline) quartiles to capture possible effects of more extreme values. Most municipality-level variables were retrieved from publicly available data from the Swiss Federal Statistical Office for 2012 and 2013. Data on religious denomination and Swiss SEP were based on the year 2000. A more detailed description of the variables is given in the online supplementary material, section 1 , figure 1-6 and table 2.

The SNVCS has received ethical committee approval. According to the Swiss Human Research Act (Art.2.2 al.c.), additional ethical committee review for this study was not required because anonymised health-related data were used.

\section{Statistical analysis}

We developed Bayesian hierarchical logistic regression models to investigate the spatial heterogeneity of HPV vaccination uptake across municipalities (table 1). First, we fitted a model that captures spatial variation at the municipality level (model 1). Spatial autocorrelation was modelled using the Besag-York-Mollié (BYM) conditional autoregressive prior distribution. ${ }^{43}$ For municipalities not sampled by the survey, BYM borrows information about the uptake from the neighbouring municipalities. Second, we added a random effect at the cantonal level (model 2) to test the hypothesis that cantons represent an important contextual factor for HPV vaccination uptake. Third, we additionally included all covariates (model 3, 'full model') and calculated the percentage of municipal variation explained by the cantonal random effect and the covariates. To do this, we calculated the median posterior variance of the municipal random effect (sum of spatially correlated and uncorrelated component) in each of these models and the percentage reduction of this variance in models 2 and 3 compared with model 1 .

To examine the effect of the prespecified covariates, we performed model selection using the deviance information criterion (DIC). ${ }^{44}$ In addition to models $1-3$, we ran four alternative models that only included the municipal random effect and the covariates (model 4), the cantonal random effect (model 5), the cantonal random effect and the covariates (model 6) and the covariates only (model 7 ). We also examined the univariable association with each of the eight covariates (model 8). We present the univariable associations (model 8), a fully adjusted logit model excluding random effects (model 7) and the model from 1 to 7 with the smallest DIC. We present results as median OR with $95 \%$ credible intervals.

We conducted three sensitivity analyses. First, we examined whether the non-respondents differ from respondents with respect to the covariates. Second, we compared results of the main analysis with models that assumed that all non-respondents were vaccinated, or that all non-respondents were not vaccinated. Third, we examined whether the survey sampling method affected the results by including sampling method as a covariate in the model with the smallest DIC.

Inference was performed using the Integrated Nested Laplace Approximation for latent Gaussian models. ${ }^{45}$ Further details about the different models, their implementation and the sensitivity analyses are provided in the online supplementary material (sections 2 and 3 ).

\section{RESULTS}

We analysed data from 21 of 26 cantons $(91.1 \%$ of the Swiss population, see online supplementary table 1).

\section{Data characteristics}

We analysed data from 8965 out of 14106 sampled girls from the participating cantons. We excluded 2056 individuals sampled in 2008, 3072 individuals who did not respond and 13 individuals with missing information about municipality of residence. The average response rate was $75.1 \%$ and ranged from $39.9 \%$ to $92.1 \%$ between cantons (see online supplementary figure 7 and table 1). Among the included participants, data on nationality were missing in $11.7 \%$ (1051/8965), concentrated in four cantons (see online supplementary material, section 1 ). The average vaccination uptake in survey participants from the 21 cantons and over all survey periods was $53.2 \%$ (95\% 


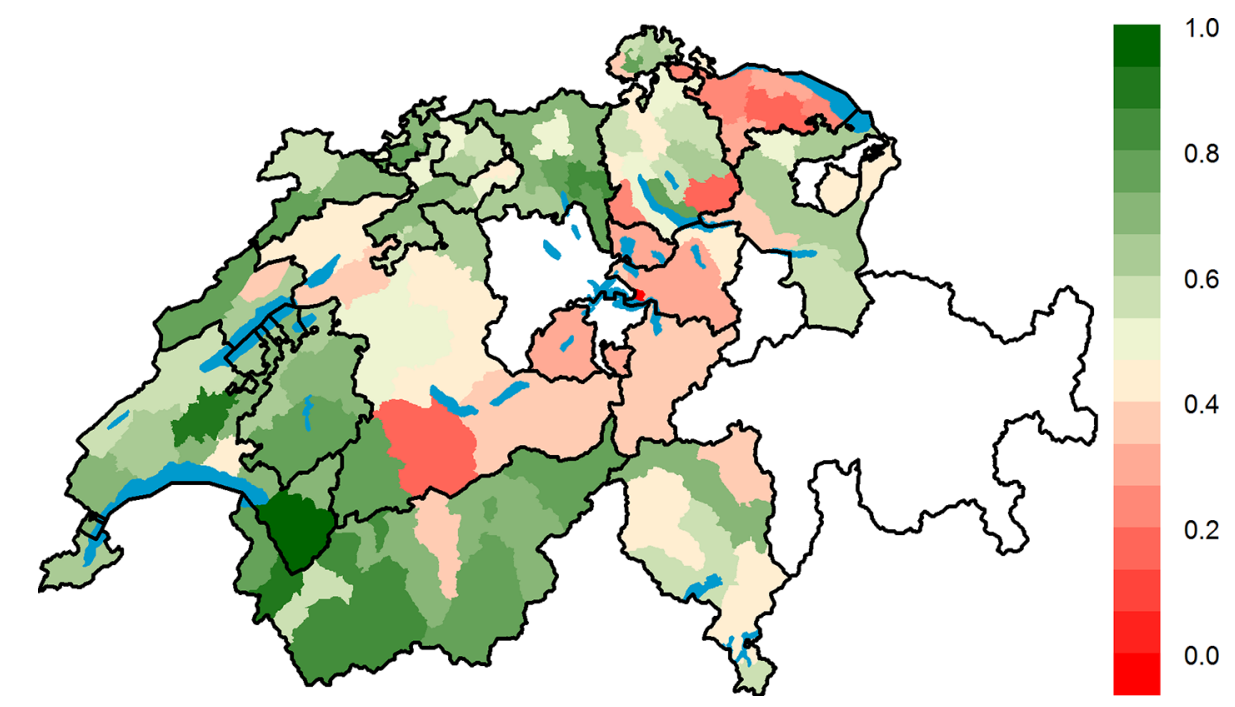

Figure 1 Crude human papillomavirus (HPV) vaccination uptake per district in Switzerland over all survey periods (2009-2016). The vaccination uptake was computed by dividing the number of girls who received at least one dose of HPV vaccine with the total number of responding girls from the corresponding district. White areas represent cantons for which we did not get authorisation to analyse the data.

CI $46.8 \%$ to $59.7 \%$ ) but varied greatly between cantons (figure 1). Seventy per cent of included girls in the survey were Swiss, $66 \%$ and $27 \%$ lived in German-speaking and French-speaking municipalities, respectively. Sixty-seven per cent of included girls lived in a canton where schoolbased vaccination was available in one or more schools (table 2).

\section{Spatial variability}

Model 1 showed considerable spatial variation of HPV uptake at the municipal level (top panel, figure 2). Including a random effect term at the cantonal level (Model 2) showed that about $63 \%$ of this variation is explained by cantonal differences (middle panel, figure 2). Additionally, including covariates further accounted for much of the spatial variation (Model 3): about $88 \%$ of the spatial variation (at the municipal level) is explained by cantonal differences and the considered covariates (bottom panel, figure 2).

\section{Model selection}

Of the fitted models $1-7$, the model with the smallest DIC was the full model (model 3, DIC=11419) (table 1). Results from the univariable models (model 8), the fully adjusted logit (model 7) and the full model (model 3) are shown in figure 3 and in more detail in the online supplementary table 3. The association of individual covariates (model 8) becomes weaker after adjusting for all covariates (model 7) and additionally including the random effects (model 3). We observed persistent strong associations for nationality, political opinion, availability of school-based HPV vaccination and survey period.

\section{Associations between vaccination uptake and covariates}

Availability of school-based vaccination delivery in a canton was strongly associated with higher vaccination uptake (figure 3 and online supplementary table 3, OR from full model 2.51; 95\% credible interval 1.77 to 3.56 ). Living in a municipality in the lowest quartile of acceptance of the referendum on the revision of the epidemic law was associated with reduced uptake (OR $0.61 ; 95 \%$ credible interval 0.50 to 0.73 ) and living in a municipality in the highest quartile was associated with increased uptake (OR 1.22; $95 \%$ credible interval 0.99 to 1.50 ). These covariates were strongly correlated; only $40 \%$ of girls living in municipalities with low acceptance of the vote lived in a canton with school-based vaccination, compared with $85 \%$ of girls from municipalities with high acceptance (see online supplementary figure 8).

Vaccination uptake was higher in municipalities in the lowest Swiss SEP quartile (OR 1.18; 95\% credible interval 1.00 to 1.38) and among non-Swiss residents (OR 1.23; $95 \%$ credible interval 1.09 to 1.39 ). Vaccination uptake increased over the three periods of the cross-sectional surveys (OR 1.22; 95\% credible interval 1.07 to 1.38 and OR $1.58 ; 95 \%$ credible interval 1.38 to 1.81 for 2011-2013 and 2014-2016, respectively). In the full model, there was no evidence that uptake differed between municipalities in high (highest quartile) and medium (second and third quartile) levels of SEP (OR highest quartile 0.93; $95 \%$ credible interval 0.78 to 1.10 ).

In univariable models, living in a French-speaking municipality was associated with higher vaccination uptake while living in a rural or protestant municipality was associated with lower uptake (figure 3 and online supplementary table 3). However, there was little evidence that these factors were associated with HPV vaccine uptake after adjusting for other covariates and accounting for cantonal and municipal-level differences (full model). Language region was highly correlated with both schoolbased vaccination (school-based vaccination was available in all French-speaking cantons) and vote results $(<1 \%$ of girls from French-speaking regions lived in municipalities 
Table 2 Characteristics of the included participants of the Swiss National Vaccination Coverage Survey on human papillomavirus

\begin{tabular}{|c|c|c|c|c|}
\hline Covariates & Proportion & Total N & $\begin{array}{l}\text { Vaccination } \\
\text { uptake } \\
\text { (proportion) }\end{array}$ & $\mathbf{N}$ \\
\hline \multicolumn{5}{|l|}{ Nationality } \\
\hline Unknown & 0.12 & 1051 & 0.66 & 690 \\
\hline Swiss & 0.70 & 6254 & 0.51 & 3199 \\
\hline Non-Swiss & 0.19 & 1660 & 0.60 & 991 \\
\hline \multicolumn{5}{|l|}{ Urbanisation levels* } \\
\hline Rural & 0.17 & 1549 & 0.49 & 758 \\
\hline Semi-urban & 0.18 & 1650 & 0.53 & 868 \\
\hline Urban & 0.64 & 5766 & 0.56 & 3254 \\
\hline \multicolumn{5}{|l|}{ SEP quartile* } \\
\hline Lowest SEP & 0.26 & 2364 & 0.59 & 1401 \\
\hline Baseline SEP & 0.53 & 4722 & 0.53 & 2504 \\
\hline Highest SEP & 0.21 & 1879 & 0.52 & 975 \\
\hline \multicolumn{5}{|l|}{ Political opinion* } \\
\hline Lowest acceptance & 0.24 & 2177 & 0.38 & 815 \\
\hline Baseline acceptance & 0.59 & 5285 & 0.59 & 3096 \\
\hline Highest acceptance & 0.17 & 1503 & 0.64 & 969 \\
\hline \multicolumn{5}{|l|}{ Religious denomination* } \\
\hline No majority & 0.46 & 4099 & 0.57 & 2326 \\
\hline$\geq 50 \%$ Protestant & 0.11 & 973 & 0.45 & 435 \\
\hline$\geq 50 \%$ Catholic & 0.43 & 3893 & 0.54 & 2119 \\
\hline \multicolumn{5}{|l|}{ Language region* } \\
\hline German speaking & 0.66 & 5941 & 0.49 & 2930 \\
\hline French speaking & 0.27 & 2437 & 0.68 & 1658 \\
\hline Italian speaking & 0.07 & 587 & 0.5 & 292 \\
\hline \multicolumn{5}{|c|}{ School-based vaccination $†$} \\
\hline No & 0.33 & 2936 & 0.37 & 1084 \\
\hline Yes & 0.67 & 6029 & 0.63 & 3796 \\
\hline \multicolumn{5}{|l|}{ Survey period $†$} \\
\hline 2009-2010 & 0.23 & 2064 & 0.46 & 945 \\
\hline $2011-2013$ & 0.42 & 3793 & 0.54 & 2039 \\
\hline 2014-2016 & 0.35 & 3108 & 0.61 & 1896 \\
\hline
\end{tabular}

${ }^{*}$ Municipality level covariate.

†Cantonal level covariate.

HPV, human papillomavirus; SEP, socioeconomic position.

with lowest acceptance of the referendum, compared with 38\% for German-speaking regions).

The results of our sensitivity analysis strengthened the validity of our findings for most of the covariates. Imputing vaccine uptake in non-respondents using extreme assumptions (all vaccinated or all non-vaccinated) did not substantially change the estimated OR for most covariates (see online supplementary material, section 3 and table 4,5). The estimated ORs did change for nationality and survey period; non-Swiss individuals were over-represented among non-respondents, and the last survey period had a higher proportion of non-respondents

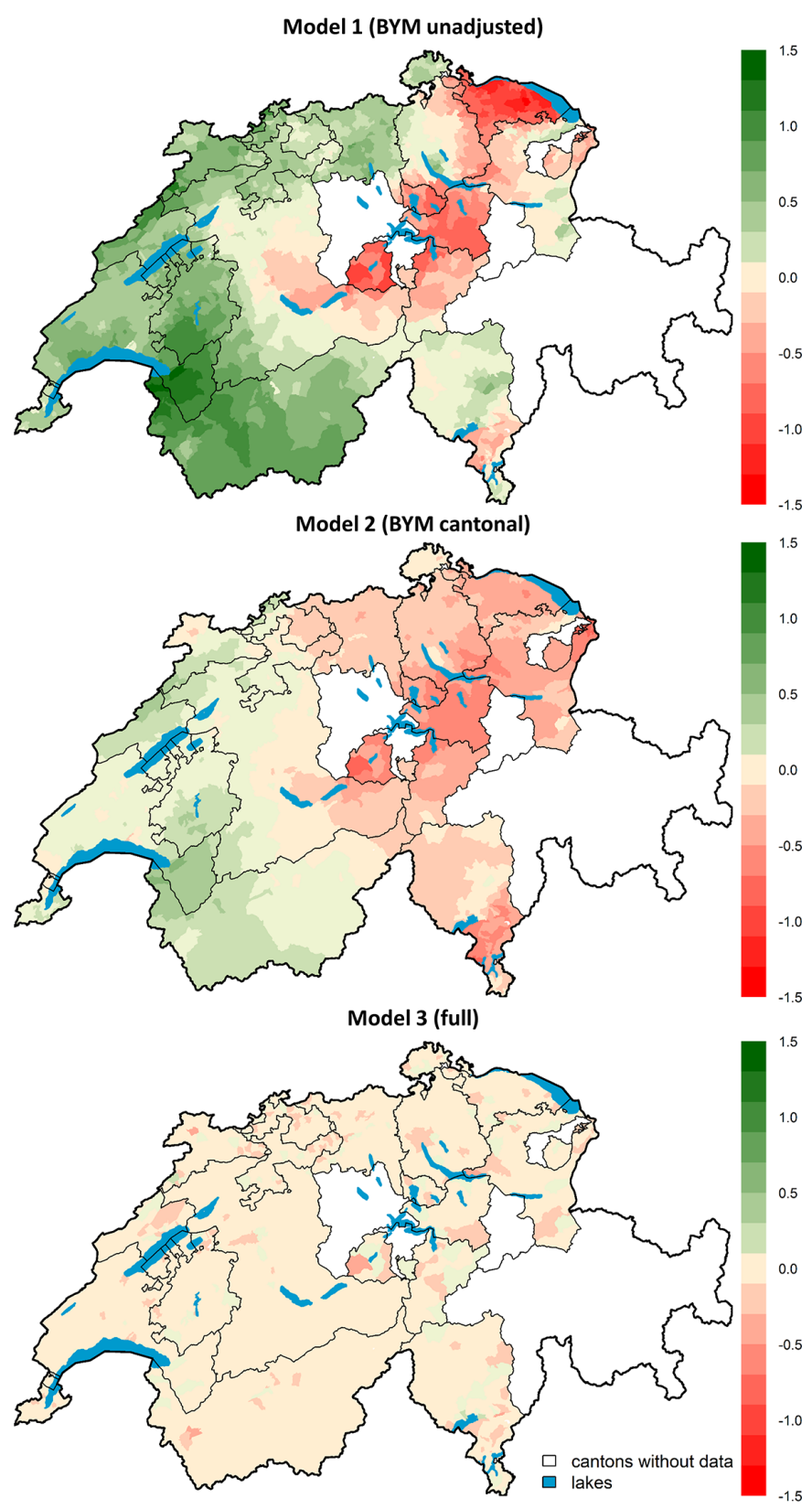

Figure 2 Spatial variation of human papillomavirus (HPV) vaccination uptake in Switzerland at the municipal level. Top panel: spatial variation accounting only for correlation between neighbouring municipalities (Model 1, Besag-YorkMollié model, BYM unadjusted); middle panel: remaining spatial variation after adjusting for cantonal differences (Model 2, BYM cantonal); bottom panel: remaining spatial variation after adjusting for cantonal differences and covariates (Model 3, full). Shown are the differences from the mean on the log odds scale. Municipalities with no information about HPV vaccination uptake borrow information from the first-order neighbouring municipalities. White areas represent cantons for which we did not get authorisation to analyse the data.

compared with the other two survey periods. For schoolbased vaccination, the ORs were slightly reduced for both extreme assumptions when compared with the full model, but remained high (OR 1.94; 95\% credible interval 1.34 


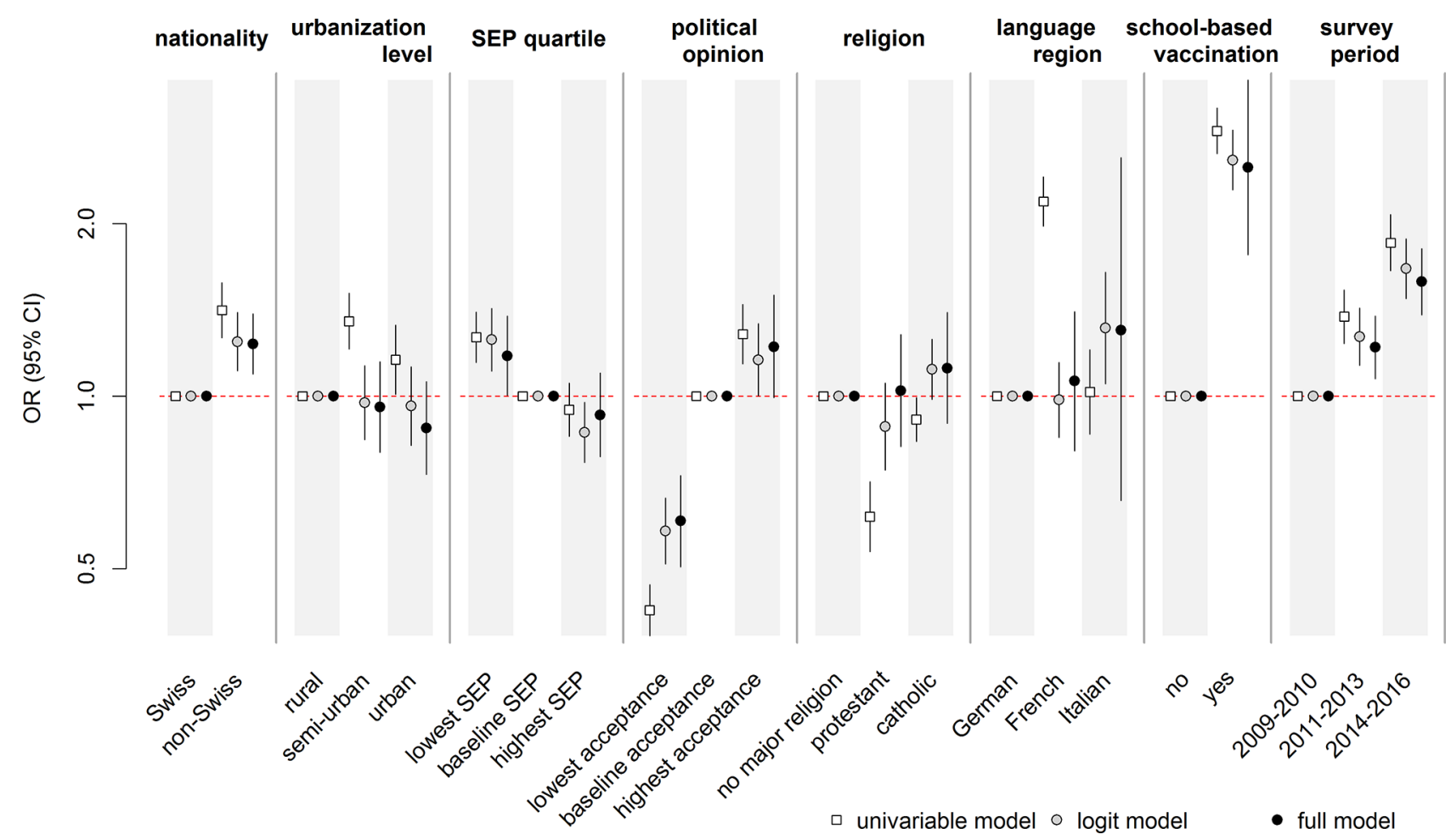

Figure 3 OR and $95 \%$ credible intervals for being vaccinated for human papillomavirus. The full model (model 3 ) is adjusted for all covariates and includes random effect terms to account for cantonal and municipal differences in uptake and spatial autocorrelation at the municipality level. The adjusted model (model 7) includes all covariates without any random effect terms. The univariable model (model 8) includes each covariate individually at a time without random effect terms.

to 2.83 and OR 2.02; $95 \%$ credible interval 1.47 to 2.81 , respectively). Accounting for different sampling methods in the full model resulted in similar estimates of OR for the covariates (see online supplementary table 5 ).

\section{DISCUSSION}

This spatial analysis of cross-sectional survey data found that cantonal differences and included covariates explained up to almost $90 \%$ of municipality-level variation in HPV vaccination uptake among girls in Switzerland. Availability of school-based vaccination delivery was strongly associated with increased HPV vaccination uptake and low municipal-level acceptance of a popular vote about the revision of the epidemic law, which included strengthening of vaccination promotion, was strongly associated with decreased uptake. Uptake of HPV vaccination increased over the three survey periods from 2009 to 2016.

The main strength of our study was the availability of survey data based on written vaccination records from cantons that included more than $90 \%$ of the Swiss population during the roll-out of HPV vaccination programmes from 2009 to 2016. We were able to include a wide range of covariates at individual, municipal and cantonal levels. Furthermore, our analysis accounted for spatial autocorrelation, which could result in inaccurate associations if ignored. ${ }^{28}{ }^{46}$ Our study has some limitations. First, the five cantons that did not participate in this study all come from the German-speaking part of Switzerland. We do not expect this to have biased our results markedly because vaccination uptake in the non-participating cantons was similar to that of other German-speaking cantons (see online supplementary table 1). Second, the survey sampling methods and response rates differed between cantons. Our sensitivity analysis showed that the effect of covariates remained similar after accounting for differences in sampling methods. Third, our findings might have been affected by selection bias. If parents of vaccinated girls were more likely to respond to the survey, HPV vaccine uptake levels might be overestimated. However, even under extreme assumptions about vaccine uptake in non-respondents, the association of the covariates with vaccination uptake remained similar (except for nationality and survey period which had large differences in response rates). Fourth, the municipal-level covariates were based on data collected in the national census from 2000, before HPV vaccination programmes began. We do not expect this to have affected our study because the composition of communities in Switzerland according to factors such as religion and SEP show little variation over time. ${ }^{47}$ Lastly, the most recent information about the organisation of cantonal HPV vaccination programmes, on which we based our analysis, was a report published in 2009. The decision to implement school-based vaccination could be taken at regional, cantonal or school health department level and aspects of the programmes in some cantons might have changed since then. The report only stated whether school-based vaccination delivery 
was available, but not in how many schools. Despite this limitation, we found this variable to be strongly associated with uptake.

To our knowledge, our study is the first analysis of spatial variation in HPV vaccination uptake in a country using outcome data at the individual level and adjusting for spatial autocorrelation. A systematic review of 25 studies of factors associated with HPV vaccine uptake, published up to 2011, found that most were cross-sectional studies from a single or limited number of states in the USA. ${ }^{14}$ The studies typically included factors at the individual level and controlled for no or a limited number of potential confounders. The review highlighted that the highest levels of vaccination uptake came from studies with schoolbased programmes, which corroborates our finding that availability of school-based vaccination delivery is associated with higher uptake. We found three studies that used spatial autocorrelation models, published since 2011, all from limited geographical areas in the USA. The first targeted uninsured and publicly insured children in North Carolina, ${ }^{17}$ the second used data from an internet survey from the Twin Cities Metropolitan Area of Minnesota ${ }^{22}$ and the third was based on seven Minnesota counties. ${ }^{16}$ In these studies, substantial spatial variation remained after adjusting for their covariates. Our study covered the majority of a whole country and the full model explained almost all small-scale spatial variation. Two studies that examined geographical variation in HPV vaccination uptake according to voting patterns, in the Netherlands ${ }^{11}$ and the USA, ${ }^{15}$ found lower HPV vaccine uptake in areas that elected religious or conservative parties. An advantage of our study is that we used voting data from a referendum that was more closely linked to people's attitudes towards vaccination than voting for a political party in general.

We found that girls living in municipalities with the lowest percentage acceptance of a vote to revise an epidemic law were less likely to be vaccinated. Some ${ }^{18202127}$ but not all ${ }^{11} 16192023-26$ studies have found an inverse association between SEP (or poverty based on income) and HPV vaccine uptake. This discrepancy between studies might be due to differences in national healthcare and health insurance systems. We found that girls living in municipalities in the lowest quartile of Swiss SEP were more likely to receive HPV vaccination than in municipalities in the middle quartiles in the univariable, but not the multivariable analysis. Our finding that non-Swiss girls were more likely to be vaccinated than Swiss girls could mean that people with family origins outside Switzerland accept vaccination more readily than Swiss people but it might also reflect household-level socioeconomic disparities that were not captured by the Swiss SEP. Since non-Swiss girls had a higher non-response rate, interpretation should be treated with caution. Ethnicity was not recorded in the SNVCS and is not routinely recorded in Switzerland. Other studies that have considered ethnicity report lower levels of HPV vaccine uptake in girls from non-white ethnic groups. ${ }^{14}$ We think that our findings about the importance of school-based vaccination delivery and attitudes towards vaccination can be applied to other high-income countries in which HPV vaccination is covered by health insurance, but where HPV vaccination coverage could be improved. The cultural, linguistic and geographic heterogeneity of the Swiss population means that it shares characteristics with a range of different settings.

The findings of our study support the hypothesis that there is interplay between people's attitudes about vaccination, the availability of vaccination services and the probability of an individual girl receiving HPV vaccination. The best-fitting model included a random effect at the cantonal level and, together with selected covariates, explained almost all small-scale spatial variation in HPV vaccine uptake. Cantons have considerable autonomy in providing health services and represent a contextual factor for vaccine accessibility. Vaccine scepticism in a community, in turn, could impact the political outcome of decision makers and hence affect vaccination policies. Our findings do not necessarily represent causal associations because of the ecological nature of the associations and the cross-sectional nature of our study design. The strong association between HPV vaccination uptake and patterns of voting about vaccination laws at the municipality level are, however, consistent with the suggestion that scepticism or opposition to vaccination could influence decisions of parents and their daughters to get vaccinated. A nationally representative Swiss survey found that fear of side effects and general opposition to vaccination were two of the main reasons that participants gave for not being vaccinated against HPV. ${ }^{48}$ The vote results were also strongly correlated with the availability of schoolbased vaccination. This relation might indicate how individuals shape their community, thereby influencing health services and affecting health outcomes. Thus, the difficulty to achieve higher levels of HPV vaccination uptake in areas with high levels of vaccine scepticism result not only from vaccine refusal, but also from a lack of easy access to vaccination such as through school-based delivery.

We conducted a multilevel spatial analysis to identify the factors that are associated with the spatial variation in HPV vaccination uptake in Switzerland. Our study highlights the importance of taking into account spatial autocorrelation and covariates at different spatial levels. Our results support the importance of an interplay between regional contextual factors and vaccine scepticism in determining HPV vaccination uptake. Our study suggests that higher levels of HPV vaccination could be achieved by efforts to mitigate vaccine scepticism, which might then permit broader use of school-based delivery of HPV vaccination.

\section{Author affiliations}

${ }^{1}$ Institute of Social and Preventive Medicine, University of Bern, Bern, Switzerland ${ }^{2}$ Graduate School for Cellular and Biomedical Sciences, University of Bern, Bern, Switzerland

${ }^{3}$ Graduate School for Health Sciences, University of Bern, Bern, Switzerland ${ }^{4}$ Epidemiology, Biostatistics and Prevention Institute, University of Zurich, Zurich, Switzerland 
${ }^{5}$ Communicable Diseases, Swiss Federal Office of Public Health, Bern, Switzerland ${ }^{6}$ Institute of Political Science, University of Bern, Bern, Switzerland ${ }^{7}$ Department of Pediatrics, Pediatric Respiratory Medicine, Inselspital, Bern University Hospital, University of Bern, Bern, Switzerland

Acknowledgements We would like to thank the cantons for providing access to their HPV vaccination survey data. We would also like to thank Dr Jan von Overbeck and Vanessa Arn for their help in obtaining agreements from the cantons.

Contributors Conceptualisation: MR, GK, CLA, BDS, NL and MB; Methodology: GK, MR, BDS, CLA and NL; Formal analysis: GK; Validation: BDS, NL, CLA, CH, PL, $A S, M B$ and MM; Writing —original draft: MR and GK; Writing — review and editing: MR, GK, CLA, BDS, NL, PL, AS, MM, MB and CH; Resources: PL, CH, AS and MM; Supervision: CLA, BDS and NL.

Funding This study was supported by the Swiss Cancer League and the Swiss Cancer Research foundation (grant No. 3049-08-2012 and 3515-082014). BDS was supported by a Swiss National Science Foundation fellowship (PZOOP3_147987).

\section{Competing interests None declared.}

Patient consent Not required.

Provenance and peer review Not commissioned; externally peer reviewed.

Data sharing statement Data are from the Swiss National Vaccination Coverage Survey whose authors may be contacted at Bundesamt für Gesundheit BAG, Abteilung Übertragbare Krankheiten, Sektion Impfempfehlungen und Bekämpfungsmassnahmen, CH-3003 Bern. Email: epi@bag.admin.ch, Tel. +4158 4638706 .

Open Access This is an Open Access article distributed in accordance with the Creative Commons Attribution Non Commercial (CC BY-NC 4.0) license, which permits others to distribute, remix, adapt, build upon this work non-commercially, and license their derivative works on different terms, provided the original work is properly cited and the use is non-commercial. See: http://creativecommons.org/ licenses/by-nc/4.0/

(c) Article author(s) (or their employer(s) unless otherwise stated in the text of the article) 2018. All rights reserved. No commercial use is permitted unless otherwise expressly granted.

\section{REFERENCES}

1. World Health Organization. Human papillomavirus (HPV) and cervical cancer. 2016 http://www.who.int/mediacentre/factsheets/fs380/en/

2. Bruni L, Diaz M, Barrionuevo-Rosas L, et al. Global estimates of human papillomavirus vaccination coverage by region and income level: a pooled analysis. Lancet Glob Health 2016;4:e453-e463.

3. Brisson M, van de Velde N, Franco EL, et al. Incremental impact of adding boys to current human papillomavirus vaccination programs: role of herd immunity. J Infect Dis 2011;204:372-6.

4. Public Health England. Human Papillomavirus (HPV) vaccination coverage in adolescent females in England: 2015/2016. 2016 https://www.gov.uk/government/uploads/system/uploads/ attachment_data/file/578729/HPV_vaccination-_2015-16.pdf (accessed 29 Jun 2017).

5. National HPV Vaccination Register. HPV Vaccination coverage 2015 - national australia hpv vaccination program register for females turning 15 years of age in 2015. 2017 http://www.hpvregister.org.au/ research/coverage-data/HPV-Vaccination-Coverage-2015.

6. Potts A, Sinka K, Love J, et al. High uptake of HPV immunisation in Scotland--perspectives on maximising uptake. Euro Surveill 2013;18:20593.

7. Giambi C, Donati S, Declich S, et al. Estimated acceptance of HPV vaccination among Italian women aged $18-26$ years. Vaccine 2011;29:8373-80.

8. Héquet $D$, Rouzier R. Determinants of geographic inequalities in HPV vaccination in the most populated region of France. PLoS One 2017;12:e0172906.

9. Poethko-Müller C, Buttmann-Schweiger N; KiGGS Study Group. [HPV vaccination coverage in German girls: results of the KiGGS study: first follow-up (KiGGS Wave 1)]. Bundesgesundheitsblatt Gesundheitsforschung Gesundheitsschutz 2014;57:869-77.

10. Durham DP, Ndeffo-Mbah ML, Skrip LA, et al. National- and statelevel impact and cost-effectiveness of nonavalent HPV vaccination in the United States. Proc Natl Acad Sci U S A 2016;113:5107-12.
11. Rondy M, van Lier A, van de Kassteele J, et al. Determinants for HPV vaccine uptake in the Netherlands: a multilevel study. Vaccine 2010;28:2070-5.

12. Holman DM, Benard V, Roland KB, et al. Barriers to human papillomavirus vaccination among US adolescents: a systematic review of the literature. JAMA Pediatr 2014;168:76-82.

13. Bühlmann M. Politische partizipation im kommunalen kontext: der einfluss lokaler kontexteigenschaften auf individuelles politisches partizipationsverhalten. Aufl. Bern: Haupt, 2006;1.

14. Kessels SJ, Marshall HS, Watson M, et al. Factors associated with HPV vaccine uptake in teenage girls: a systematic review. Vaccine 2012;30:3546-56.

15. Bernstein S, North A, Schwartz J, et al. State-level voting patterns and adolescent vaccination coverage in the united states, 2014. Am J Public Health 2016;106:1879-81.

16. Finney Rutten LJ, Wilson PM, Jacobson DJ, et al. A populationbased study of sociodemographic and geographic variation in HPV vaccination. Cancer Epidemiol Biomarkers Prev 2017;26:533-40.

17. Trogdon JG, Ahn T. Geospatial patterns in human papillomavirus vaccination uptake: evidence from uninsured and publicly insured children in North Carolina. Cancer Epidemiol Biomarkers Prev 2015;24:595-602.

18. Henry KA, Stroup AM, Warner EL, et al. Geographic factors and human papillomavirus (hpv) vaccination initiation among adolescent girls in the united states. Cancer Epidemiol Biomarkers Prev 2016;25:309-17.

19. Guthmann JP, Pelat C, Célant N, et al. Socioeconomic inequalities to accessing vaccination against human papillomavirus in france: results of the health, health care and insurance survey, 2012. Rev Epidemiol Sante Publique 2017;65:109-17.

20. Pruitt SL, Schootman M. Geographic disparity, area poverty, and human papillomavirus vaccination. Am J Prev Med 2010;38:525-33.

21. Tsui J, Gee GC, Rodriguez HP, et al. Exploring the role of neighborhood socio-demographic factors on HPV vaccine initiation among low-income, ethnic minority girls. J Immigr Minor Health 2013;15:732-40.

22. Nelson EJ, Hughes J, Oakes JM, et al. Geospatial patterns of human papillomavirus vaccine uptake in Minnesota. BMJ Open 2015;5:e008617.

23. Lefevere E, Hens N, De Smet F, et al. Dynamics of HPV vaccination initiation in Flanders (Belgium) 2007-2009: a Cox regression model. BMC Public Health 2011;11:470.

24. Smith LM, Brassard P, Kwong JC, et al. Factors associated with initiation and completion of the quadrivalent human papillomavirus vaccine series in an Ontario cohort of grade 8 girls. BMC Public Health 2011;11:645.

25. Schülein S, Taylor KJ, König J, et al. Factors influencing uptake of HPV vaccination among girls in Germany. BMC Public Health 2016;16:995.

26. Centers for Disease Control and Prevention (CDC). National, state, and local area vaccination coverage among adolescents aged 13-17 years --- United States, 2009. MMWR Morb Mortal Wkly Rep 2010;59:1018-23.

27. Gerend MA, Weibley E, Bland H. Parental response to human papillomavirus vaccine availability: uptake and intentions. J Adolesc Health 2009;45:528-31.

28. Langford IH, Leyland $\mathrm{AH}$, Rasbash J, et al. Multilevel modelling of the geographical distributions of diseases. J R Stat Soc Ser C Appl Stat 1999;48:253-68.

29. Bundesamt für Gesundheit. Vaccination contre le cancer $d u$ col de l'utérus- début des programmes cantonaux de vaccination, 2008.

30. Spaar A, Masserey V. HPV-Impfung in der Schweiz. Impfraten und aktuelle Daten zu Wirksamkeit und Sicherheit. Pädiatrie 2015;6:8-11.

31. Bundesamt für Gesundheit. Die HPV-impfprogramme in der schweiz: eine synthese von 2007 bis 2010. Bulletin 2010;43:949-53.

32. EBPI- Universität Zürich, Bundesamt für Gesundheit. Durchimpfung von 2-, 8- und 16- Jährigen Kindern in der Schweiz, 1999-2016. http://www.bag.admin.ch/themen/medizin/00682/00685/02133/ index.html? lang=de (accessed 9 Dec 2017).

33. Bundesamt für Gesundheit. Durchimpfung von 2-,8- und 16-Jährigen in der Schweiz, 2011 bis 2013. Bulletin 2015;28.

34. Lang P, Zimmermann H, Piller U, et al. The swiss national vaccination coverage survey, 2005-2007. Public Health Rep 2011;126(Suppl 2):97-108.

35. Bundesamt für Statistik. Statistik der bevölkerung und der haushalte (STATPOP), sektion demografie und migration. ständige nichtständige wohnbevölk. nach institutionellen gliederungen staatsangehör. Kateg. Geschlecht Alter. 2013 https://www.bfs.admin ch/bfs/de/home/statistiken/bevoelkerung/migration-integration/ auslaendische-bevoelkerung.assetdetail.3262116.html (accessed 12 Sep 2017). 
36. PLANeS. Schweizerische stiftung für sexuelle un reproduktive gesundheit. evaluation kantonaler hpv-impfprogramme, definitiver bericht für das BAG, 2009.

37. Bundesamt für Statisik. Die raumgliederungen der schweiz 2016 - ms-excel version. bundesamt für statistik, schweizerische eidgenossenschaft 2017. 2017 https://www.bfs.admin.ch/bfs/de/ home/grundlagen/raumgliederungen.assetdetail.2118475.html (accessed 21 Sep 2017)

38. Bundesamt für Statistik. Swiss population census 2000. Bern, Schweiz, 2000. https://www.bfs.admin.ch/bfs/en/home/statistics/ population/surveys/census.html.

39. Panczak R, Galobardes B, Voorpostel M, et al. A Swiss neighbourhood index of socioeconomic position: development and association with mortality. J Epidemiol Community Health 2012;66:1129-36.

40. Spycher BD, Feller M, Röösli M, et al. Childhood cancer and residential exposure to highways: a nationwide cohort study. Eur $J$ Epidemiol 2015;30:1263-75.

41. Chancellery F. Loi fédérale sur la lutte contre les maladies transmissibles de l'homme (Loi sur les épidémies, LEp). https://www. admin.ch/ch/f/pore/rf/cr/2007/20071012.html (accessed 19 May 2017).
42. Bundesamt für Statistik. Statistik der wahlen und abstimmungen, abstimmungen 2013: epidemiengesetz. neuch\&acirc;tel, suisse. 2013 https://www.bfs.admin.ch/bfs/de/home/statistiken/politik/ erhebungen/swa.html (accessed 8 Aug 2017).

43. Besag J, York J, Molli A. Bayesian image restoration, with two applications in spatial statistics. Ann Inst Stat Math 1991;43:1-20.

44. Spiegelhalter DJ, Best NG, Carlin BP, et al. Bayesian measures of model complexity and fit. Journal of the Royal Statistical Society: Series B 2002;64:583-639.

45. Rue H, Martino S, Chopin N. Approximate bayesian inference for latent gaussian models by using integrated nested laplace approximations. Journal of the Royal Statistical Society: Series B 2009;71:319-92.

46. Werneck GL, Maguire JH. Spatial modeling using mixed models: an ecologic study of visceral leishmaniasis in Teresina, Piauí State, Brazil. Cadernos de Saúde Pública 2002;18:633-7.

47. Bundesamt für Statistik. Religions. Religionen. https://www.bfs. admin.ch/bfs/fr/home/statistiques/population/langues-religions/ religions.html (accessed 5 Sep 2017).

48. Bundesamt für Gesundheit, Abteilung übertragbare Krankheiten. Die HPV-impfung in der schweiz: resulate einer nationalen befragung im jahr 2014. Bulletin 2015;23/15:445-52. 